\title{
Analyzing the Performance Limits of Articulated Soft Robots based on the ESPi Framework: Applications to Damping and Impedance Control
}

\author{
Manuel Keppler ${ }^{1}$, Florian Loeff ${ }^{1}$, David Wandinger ${ }^{1}$, and Clara Raschel ${ }^{1}$, and Christian Ott ${ }^{1}$
}

\begin{abstract}
In situations of harsh impacts, damping injection directly on the link of an articulated soft robot is challenging and usually requires high actuator torques at the moment of impact. In this work, we discuss the underlying reasons and analyze the performance limitations arising in the implementation of basic impedance elements, such as springs and dampers, through the elastic structure preserving impedance (ESPi) control framework. Using the insights obtained, we present a way to design impedance controllers with a damping design based on dynamic extensions. Inspired by the design of shock absorbers and the muscle-tendon model, the presented damping layout requires substantially smaller actuator torques in situations where the robot is subject to harsh impacts. The implementation is facilitated through the ESPi control framework resulting in a physically intuitive impedance design. The resulting closed-loop system can be interpreted as an interconnection of passive Euler Lagrange systems, which again, yields a passive system. The design's passive nature ensures stability in the free motion case and enables the robot to interact robustly and safely with its environment. The work focuses on robotic systems with no inertial coupling between the motor and link dynamics. Experimental results, obtained with the presented design on a dedicated series elastic actuator (SEA) test bed, are reported and discussed.
\end{abstract}

Index Terms-Compliance and Impedance Control, Compliant Joints and Mechanisms

\section{INTRODUCTION}

$\mathbf{T}$ HE safe and controlled interaction of a robotic system with its environment plays an ever-increasing role. As pointed out by Hogan [1], classic position and force control are inadequate for physical interaction tasks since they are insufficient to control the work exchanged between a manipulator and its environment. In contrast, impedance-based techniques focusing on the mechanical energy exchanged between a robot and its environment, are excellent candidates when interaction with the physical world is at the center. They enable to modulate and control the dynamic behavior of the robot while simultaneously commanding a desired position.

In [2], we introduced the elastic structure preserving impedance (ESPi) framework that enables the direct adoption of impedance control techniques to underactuated articulated soft robots (ASRs) [3]-[6]. This adoption is facilitated by establishing a link-side power port, $\left(\overline{\boldsymbol{u}}_{1}, \dot{\boldsymbol{q}}_{u}\right)$, that contains a

This project has received funding from the European Research Council (ERC) under the European Union's Horizon 2020 research and innovation programme (grant agreement No. 819358).

${ }^{1}$ The authors are with the Institute of Robotics and Mechatronics, German Aerospace Center (DLR), 82234 Oberpfaffenhofen, Germany, corresponding author: Manuel Keppler (manuel.keppler@dlr.de) virtual link-side control input, $\overline{\boldsymbol{u}}_{1}$, at the non collocated output (link position), $\dot{\boldsymbol{q}}_{u}$. Experimental results are reported in [2], [7] and shown here: https://youtu.be/sbhiNNIxMNQ

Situations may arise, however, where an ASR is subject to hard and/or fast impacts, and in these scenarios, the implementation of a damping term directly on the link can be challenging since the actuators easily run into torque saturations at the moment of impact. Motivated by this observation, we analyze the performance limits of ASRs concerning the implementation of basic impedance elements such as springs and dampers through the ESPi framework. Based on the insights gained, we present enhanced damping designs, based on dynamic extensions, which significantly reduce the risk of input saturation in the moment of a harsh impact. The inspiration for these damping designs was drawn from shock absorbers and the muscle-tendon system, which are required to showcase a smooth force variation at the moment of impact. Dynamic extension is a familiar concept in control. In [8]-[10] dynamic extensions were used to create artificial damping without the need for velocity measurements. Further, [9], [10] report elegant solutions to deal with actuator torque limits. However, such dynamic extensions have not yet been used in the context of enhancing the damping behavior of an ASR. In fact, the gain design required for our purpose is diametrical to what is recommended in [10], [11]. Roughly speaking, in [9], [10], and for the implementation of ,dirty derivatives" (filtered derivatives) [8], the spring that decouples the damper is usually chosen as stiff as possible. However, for reducing the control effort during impacts the opposite is favorable as argued in this work. The work [12] reports a tracking controller for ASRs using a filtered derivative to avoid feedback of the link jerk signals. In contrast to [12], the proposed controllers preserve the intrinsic system structure and result in a physically intuitive closed-loop behavior. In fact, the resulting closed-loop systems can be interpreted as the negative feedback interconnection of two passive Euler Lagrange systems, which again, results in a passive system. A physically motivated design allows the operator to anticipate the robot's interaction behavior with the environment (i.e. another physical system). Loosely speaking, the interaction between two physical systems that are visualizable in terms of basic mechanical elements (springs, masses, dampers), is easier to comprehend and anticipate than the interaction between a set of differential equations and the environment. This fact can hardly be overestimated when it comes to the commissioning stage since physical intuition is of immense value for tuning 
the controller. In addition, having a physically intuitive closedloop behavior provides - to some extent - a feeling for the extent of system shaping imposed by a particular controller choice. The concept imposes no limitations on the achievable closed-loop stiffness and, in particular, it can be increased above the system's intrinsic stiffness. However, on a real system with motor saturation, limits obviously exist.

The paper is organized as follows. In Section II] we establish a link-side interconnection port. Section III discusses the challenges of link-side damping injection. In Section IV we analyze the performance limits of ASRs based on the ESPi framework. Based on the insights, enhanced damping designs are presented in Section V. Section VI concludes this work with a presentation and discussion of experimental results that have been obtained on a SEA test bed.

\section{A. Model Assumptions}

Throughout this work, we consider ASRs satisfying the assumptions introduced in [13], i.e. the angular part of the kinetic energy of each rotor can be considered due only to its own rotation. Let $\boldsymbol{q} \triangleq\left[\boldsymbol{q}_{u}^{\mathrm{T}}, \boldsymbol{q}_{a}^{\mathrm{T}}\right]^{\mathrm{T}}, \boldsymbol{q}_{u}, \boldsymbol{q}_{a} \in \mathbb{R}^{n}$ be the generalized coordinates representing the unactuated link and actuated motor angles, respectively 1 The control inputs are the actuator torques $\boldsymbol{u} \in \mathbb{R}^{n}$. The kinetic and potential energies are given by $\mathcal{T}\left(\boldsymbol{q}_{u}, \dot{\boldsymbol{q}}\right) \triangleq \frac{1}{2} \dot{\boldsymbol{q}}^{\mathrm{T}} \mathcal{M}\left(\boldsymbol{q}_{u}\right) \dot{\boldsymbol{q}}$ and $\mathcal{V}(\boldsymbol{q}) \triangleq \frac{1}{2} \boldsymbol{q}^{\mathrm{T}} \mathcal{K} \boldsymbol{q}+\mathcal{V}_{g}\left(\boldsymbol{q}_{u}\right)$, with symmetric and positive definite stiffness and generalized inertia matrices

$$
\mathcal{K} \triangleq\left[\begin{array}{cc}
\boldsymbol{K} & -\boldsymbol{K} \\
-\boldsymbol{K} & \boldsymbol{K}
\end{array}\right], \quad \mathcal{M}\left(\boldsymbol{q}_{u}\right) \triangleq\left[\begin{array}{cc}
\boldsymbol{M}\left(\boldsymbol{q}_{u}\right) & \mathbf{0} \\
\mathbf{0} & \boldsymbol{B}
\end{array}\right],
$$

where $\boldsymbol{M}\left(\boldsymbol{q}_{u}\right)$ is the generalized link-side inertia matrix, $\boldsymbol{B}=\operatorname{diag}\left(b_{1}, \ldots, b_{n}\right)$ is a constant diagonal matrix of the reflected motor inertias, $\boldsymbol{K}=\operatorname{diag}\left(k_{1}, \ldots, k_{n}\right)$ is a constant diagonal matrix containing the joint stiffness values and $\mathcal{V}_{g}\left(\boldsymbol{q}_{u}\right)$ is the gravitational potential energy. The total energy is given by:

$$
\mathcal{H}=\frac{1}{2} \dot{\boldsymbol{q}}^{\mathrm{T}} \mathcal{M}\left(\boldsymbol{q}_{u}\right) \dot{\boldsymbol{q}}+\frac{1}{2} \boldsymbol{q}^{\mathrm{T}} \mathcal{K} \boldsymbol{q}+\mathcal{V}_{g}\left(\boldsymbol{q}_{u}\right)
$$

Assuming external generalized forces $\mathcal{Q} \triangleq\left[\mathcal{Q}_{1}^{\mathrm{T}}, \mathcal{Q}_{2}^{\mathrm{T}}\right]^{\mathrm{T}}$, $\mathcal{Q}_{1}, \mathcal{Q}_{2} \in \mathbb{R}^{n}$, the corresponding equations of motion are:

$$
\Sigma_{q}: \mathcal{M}\left(\boldsymbol{q}_{u}\right) \ddot{\boldsymbol{q}}+\mathcal{C}\left(\boldsymbol{q}_{u}, \dot{\boldsymbol{q}}_{u}\right) \dot{\boldsymbol{q}}+\mathcal{K} \boldsymbol{q}+\boldsymbol{G}\left(\boldsymbol{q}_{u}\right)=\left[\begin{array}{l}
\mathbf{0} \\
\boldsymbol{u}
\end{array}\right]+\mathcal{Q},
$$

with $\boldsymbol{G}\left(\boldsymbol{q}_{u}\right) \triangleq\left[\boldsymbol{g}\left(\boldsymbol{q}_{u}\right)^{\mathrm{T}}, \mathbf{0}\right]^{\mathrm{T}}=\frac{\partial \mathcal{V}_{g}\left(\boldsymbol{q}_{u}\right)}{\partial \boldsymbol{q}}$ and the Coriolis/centrifugal matrix $\mathcal{C}\left(\boldsymbol{q}_{u}, \dot{\boldsymbol{q}}_{u}\right) \triangleq \operatorname{diag}\left(\boldsymbol{C}\left(\boldsymbol{q}_{u}, \dot{\boldsymbol{q}}_{u}\right), \mathbf{0}\right)$, which is formulated such that the skew-symmetry $\dot{\mathcal{M}}\left(\boldsymbol{q}_{u}\right)=\mathcal{C}\left(\boldsymbol{q}_{u}, \dot{\boldsymbol{q}}_{u}\right)+\mathcal{C}\left(\boldsymbol{q}_{u}, \dot{\boldsymbol{q}}_{u}\right)^{\mathrm{T}}$ holds [14], [15]. For the generalized elastic forces, we use the abbreviation $\boldsymbol{\psi}(\boldsymbol{q})=\boldsymbol{K}\left(\boldsymbol{q}_{a}-\boldsymbol{q}_{u}\right)$. It is well known that ASRs define a passive operator from the applied actuator torques to motor shaft velocities, though it is not passive from the applied actuator torques to the link velocities [16].

\footnotetext{
${ }^{1}$ Throughout the text, the following indices are used: $u \ldots$ unactuated $a \ldots$ actuated, $v \ldots$ virtual, $c \ldots$ controller states due to dynamic extension.
}

\section{Shaping the Interaction With the Physical WORLD}

The ESPi framework [7] allows us to establish a link-side interconnection port which will play a central role for the implementation of impedance control concepts throughout this work. Introducing virtual motor coordinates $\boldsymbol{q}_{v} \in \mathbb{R}^{n}$ and virtual inputs $\overline{\boldsymbol{u}}_{1}, \overline{\boldsymbol{u}}_{2} \in \mathbb{R}^{n}$ such that

$$
\begin{aligned}
\boldsymbol{q}_{a} & =\boldsymbol{q}_{v}+\boldsymbol{K}^{-1} \overline{\boldsymbol{u}}_{1}, \\
\boldsymbol{u} & =\boldsymbol{B} \boldsymbol{K}^{-1} \ddot{\overline{\boldsymbol{u}}}_{1}+\overline{\boldsymbol{u}}_{1}+\overline{\boldsymbol{u}}_{2},
\end{aligned}
$$

and applying these two transformations to system $\Sigma_{q}$ yields:

$$
\Sigma_{\bar{q}}: \mathcal{M}\left(\boldsymbol{q}_{u}\right) \ddot{\overline{\boldsymbol{q}}}+\mathcal{C}\left(\boldsymbol{q}_{u}, \dot{\boldsymbol{q}}_{u}\right) \dot{\overline{\boldsymbol{q}}}+\mathcal{K} \overline{\boldsymbol{q}}+\boldsymbol{G}\left(\boldsymbol{q}_{u}\right)=\overline{\boldsymbol{u}}+\mathcal{Q},
$$

with the new generalized coordinates $\overline{\boldsymbol{q}} \triangleq\left[\boldsymbol{q}_{u}^{\mathrm{T}}, \boldsymbol{q}_{v}^{\mathrm{T}}\right]^{\mathrm{T}}$ and input vector $\overline{\boldsymbol{u}} \triangleq\left[\overline{\boldsymbol{u}}_{1}^{\mathrm{T}}, \overline{\boldsymbol{u}}_{2}^{\mathrm{T}}\right]^{\mathrm{T}}$. We refrain from providing an extensive proof, since (5) is a direct result of applying the methodology introduced in [7] for the special case of linear springs (replace $\boldsymbol{n}$ in eq. (13) of [7] with $\overline{\boldsymbol{u}}_{1}$ ). It is also straightforward to confirm by substituting (3)-(4) into (2) and observing the equivalence with (5). Comparing (2) with (5), it is clear that both equations of motion are characterized by the same Lagrangian $\mathcal{L}$, and thus the structure of the equations of motion is entirely preserved under the transforming equations (3) and (4). The only difference being: for $\Sigma_{\bar{q}}$ the Lagrangian is evaluated in terms of the $\overline{\boldsymbol{q}}$ 's, whereas for $\Sigma_{q}$ the Lagrangian is evaluated in terms of the $\boldsymbol{q}$ 's. Figure 1 compares the original and the transformed system. Intuitively, we can think of the virtual motor coordinates $\boldsymbol{q}_{v}$ being formed by shifting the original motor coordinates $\boldsymbol{q}_{a}$. The magnitude of this shift is direct proportional to the input $\overline{\boldsymbol{u}}_{1}$ with the factor of proportionality being the joint compliance $\boldsymbol{K}^{-1}$. Note that (3)-(4) comprise $n$ independen $t^{2}$ scalar equations each since, both, the stiffness and motor inertia matrix are diagonal. The following assumption guarantees boundedness of $\boldsymbol{u}$.

Assumption 1. The virtual link-side inputs $\overline{\boldsymbol{u}}_{1}$ are sufficiently smooth such that $\dot{\overline{\boldsymbol{u}}}_{1}$ exists and that $\ddot{\overline{\boldsymbol{u}}}_{1}$ is bounded.

Remark. The smoothness constraint on the virtual input defined in Assumption 1 is not specific to the ESPi framework, indeed, it is a manifestation of the physical fact that the link-side torque bandwidth of ASRs is fundamentally reduced compared to directly actuated systems.

A closer examination of the input transformation (4) reveals an interesting connection to the natural frequencies of the individual mass-spring systems which are constituted by the motor inertias and joint springs. Introducing $\omega_{n, i} \triangleq \sqrt{k_{i} / b_{i}}$, allows us to rewrite the input transformation (4) as:

$$
\boldsymbol{u}=\boldsymbol{\Omega}^{-2} \ddot{\overline{\boldsymbol{u}}}_{1}+\overline{\boldsymbol{u}}_{1}+\overline{\boldsymbol{u}}_{2},
$$

with $\boldsymbol{\Omega}^{-2} \triangleq \boldsymbol{B} \boldsymbol{K}^{-1}=\operatorname{diag}\left(\omega_{n, 1}^{-2}, \ldots, \omega_{n, n}^{-2}\right)$. The input transformation (4) has important implications for the design of elastic robots, since it reveals the fundamental connection between the system's inherent natural eigenfrequencies $\omega_{i}$ and

\footnotetext{
${ }^{2}$ Independent in the sense that each of the $n$ scalar state and input transformation equations can be solved separately.
} 


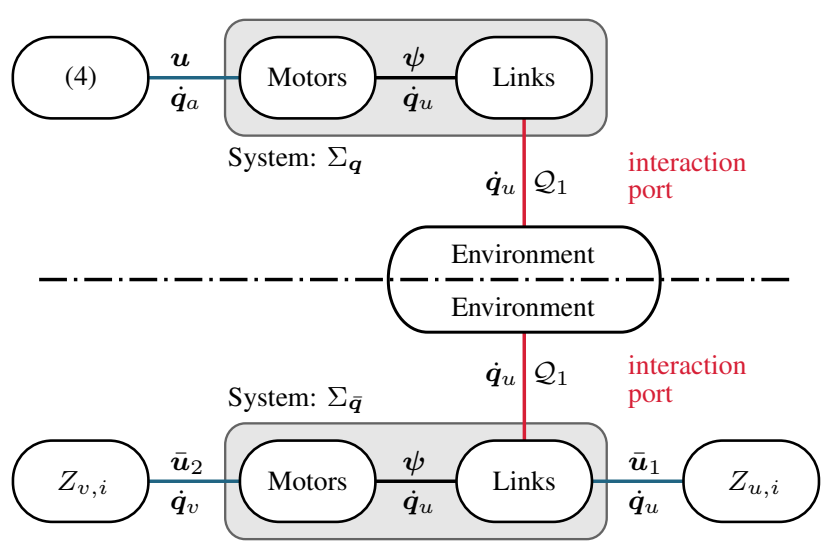

Fig. 1. The structure of the original and the transformed system. The controller interconnected with the physical system, realizes the interconnected link- and motor-side impedances on the virtual system.

the achievable link-side torque control bandwidth. This matter is discussed in detailed in Section IV

The interaction of an ASR with its environment is characterized by the way energy is exchanged. This energy exchange can be computed by taking the time derivative of the robot's total energy $\mathcal{H}$. For the system $\Sigma_{q}$, we obtain $\dot{\mathcal{H}}(\boldsymbol{q}, \dot{\boldsymbol{q}})=\dot{\boldsymbol{q}}^{\mathrm{T}}\left(\left[\mathbf{0}, \boldsymbol{I}_{n}\right]^{\mathrm{T}} \boldsymbol{u}+\mathcal{Q}_{\mathrm{ext}}\right)$ and time integration from 0 to $T$ establishes the key energy balance equation

$$
\begin{aligned}
& \mathcal{H}(\boldsymbol{q}(T), \dot{\boldsymbol{q}}(T))-\mathcal{H}(\boldsymbol{q}(0), \dot{\boldsymbol{q}}(0))= \\
& \underbrace{\int_{0}^{T}\left(\dot{\boldsymbol{q}}_{u}^{\mathrm{T}} \mathcal{Q}_{1}\right) d s}_{\text {natural exchange }}+\underbrace{\int_{0}^{T} \dot{\boldsymbol{q}}_{a}^{\mathrm{T}} \boldsymbol{u} d s}_{\text {supplied }} .
\end{aligned}
$$

Proceeding mutatis mutandis for the virtual system $\Sigma_{\bar{q}}$, we obtain $\dot{\mathcal{H}}(\overline{\boldsymbol{q}}, \dot{\overline{\boldsymbol{q}}})=\dot{\overline{\boldsymbol{q}}}^{\mathrm{T}}\left(\overline{\boldsymbol{u}}+\mathcal{Q}_{\text {ext }}\right)$ and

$$
\begin{aligned}
& \mathcal{H}(\overline{\boldsymbol{q}}(T), \dot{\overline{\boldsymbol{q}}}(T))-\mathcal{H}(\overline{\boldsymbol{q}}(0), \dot{\overline{\boldsymbol{q}}}(0))= \\
& \underbrace{\int_{0}^{T}\left(\dot{\boldsymbol{q}}_{u}^{\mathrm{T}} \mathcal{Q}_{1}\right) d s}_{\text {natural exchange }}+\underbrace{\int_{0}^{T}\left(\dot{\boldsymbol{q}}_{u}^{\mathrm{T}} \overline{\boldsymbol{u}}_{1}+\dot{\boldsymbol{q}}_{v}^{\mathrm{T}} \overline{\boldsymbol{u}}_{2}\right) d s}_{\text {supplied }},
\end{aligned}
$$

respectively. In either case, we can identify two types of power flows, see also Figure 1. The first terms on the RHS of (7) and (8) denote the respective system's natural exchange of energy with its environment. The second term on the RHS represents the artificial supply rate imposed by a particular controller. In conclusion, the Hamiltonian $\mathcal{H}$ suits as a storage function to show that $\Sigma_{\bar{q}}$ defines a passive operator $\overline{\boldsymbol{u}} \mapsto \overline{\boldsymbol{q}}$. Critically, the key port $\dot{\boldsymbol{q}}_{u}^{\mathrm{T}} \mathcal{Q}_{1}$ is preserved under the transformations (3)-(4). From the physical point of view, this port behavior determines the interaction behavior of the robot with its environment. Exploiting the link-side interconnection port, $\overline{\boldsymbol{u}}_{1}, \dot{\boldsymbol{q}}_{u}$, to interconnect impedance elements, we can shape the interaction behavior. Throughout this paper, $Z_{u, i}$ and $Z_{v, i}$ denote impedances interconnected with the $i$ th link and virtual motor, respectively, c.f. Figure 1 . The control structure that results from such interconnections is shown in Figure 2

An interesting aspect of impedance control is the superposition principle, which facilitates the understanding of complex interconnected systems.

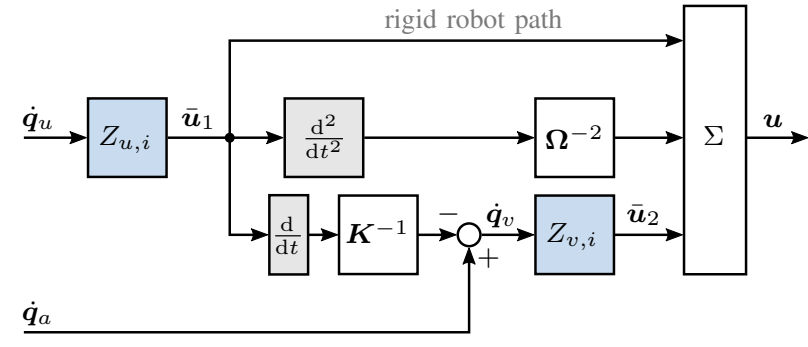

Fig. 2. Control structure for interconnecting an ASR with impedances.

\section{Challenges OF LiNK-SIDE DAMPING INJECTION}

In this section, we discuss the challenges regarding direct link-side damping injection on ASR. Based on the insights obtained, we formulate enhanced damping designs in Section IV The challenges arise from the fact that implementation of a damping term, that acts directly on a link, requires feedback of the link jerks. This fact is easily revealed within the ESPi control framework. We can use input $\overline{\boldsymbol{u}}_{1}$ to interconnect a viscous damper with each link

$$
\bar{u}_{1, i}=-d_{q, i} \dot{q}_{u, i},
$$

where $d_{q, i}$ is the damping coefficient for the $i$ th link. Making the substitutions (4) and (9), it is clear that the implementation requires feedback of the jerk signals $q_{u, i}^{(3)}$. A fundamental property of the model (2) is that these quantities can be derived from the dynamics equations without numerical differentiation, see Appendix. However, (15) reveals that the link jerk magnitudes are related to the rate of change of the external forces $\mathcal{Q}_{1}$ and, in fact, grow unbounded for impact forces approaching a step-like variation. As a consequence, the feedback of jerk signals can potentially lead to input saturation during harsh impacts with the environment. Notice that deactivation of active damping during impacts is no viable option, as it would require non-smooth joint torque variations which are physically impossible. Unless the system is at rest, such switching would cause a non-smooth variation of $\overline{\boldsymbol{u}}_{1}$, which would violate Assumption 1. What defines a harsh impact? Let us assume a sine-like force excitation of constant amplitude $A$ and frequency $\omega$ on the $i$ th joint of the form $\mathcal{Q}_{1, i}(t)=A \sin (\omega t)$, then the magnitude of $\dot{\mathcal{Q}}_{1, i}$ rises linearly with $\omega$. Hence, for the limit case $\omega \rightarrow \infty$, we have $\dot{\mathcal{Q}}_{1, i} \rightarrow \infty$ and, thus, $q_{u, i}^{(3)} \rightarrow \infty$. In conclusion, in impact situations where the impact force signal contains substantial high frequency content, the implementation of direct link damping requires large actuator torques. Note that jerk signals are contained in the feedback terms $\ddot{\bar{u}}_{1, i}$ which are scaled with $\omega_{n, i}^{-2}$. We conclude, the softer the joint, the harder the challenge of direct damping injection on the links. In practice, the "harshness" of an impact depends significantly on the relative speed and hardness of the collision partners.

It is important to notice that the challenge regarding linkside damping injection does not arise from limitations of the ESPi framework, we used for analysis here. In fact, these limitations are a manifestation of the mechanical bandwidth limitation of an elastic joint [3]. To support this statement, let us consider one of the most straightforward 
ways to implement a link-side damping term of the form (9). Implementation with a classic joint torque tracking controlle ${ }^{3}$ [17] $\boldsymbol{u}=\boldsymbol{\Omega}^{-2} \ddot{\overline{\boldsymbol{u}}}_{1}+\overline{\boldsymbol{u}}_{1}-\boldsymbol{\Omega}^{-2}\left(\boldsymbol{D}_{e} \dot{\boldsymbol{e}}+\boldsymbol{K}_{e} \boldsymbol{e}\right)+\boldsymbol{B} \ddot{\boldsymbol{q}}$, with $\boldsymbol{e} \triangleq \boldsymbol{\psi}-\overline{\boldsymbol{u}}_{1}$, reveals the feedback necessity of the link jerks through the same term, $\Omega^{-2} \ddot{\overline{\boldsymbol{u}}}_{1}$, as the damping implementation via (4). We conclude, that in order to reduce the actuator torque requirements in situations of harsh impacts, we have to avoid link jerk signals in the feedback path.

\section{Performance Limitations}

Let us analyze the actuator torques required for interconnecting basic link impedances through the link-side port established above. The insights obtained are crucial for the layout of the enhanced damping designs presented in Section V Since the transformation equations (3) and (4) are constituted by $n$ independent scalar equations, the performance analysis for a multi-joint manipulator can be conducted by analyzing each joint individually. Setting $\overline{\boldsymbol{u}}_{2}=\mathbf{0}$ and performing a Laplace transform on the input transformation (4) yields:

$$
U_{i}=\bar{U}_{i}+\left(\omega_{n, i}^{-1} s\right)^{2} \bar{U}_{i}
$$

which expresses the $i$ th motor torque in terms of the $i$ th linkside input. The first, $\bar{U}_{i}$, is the torque transmitted through the elastic element to the link. The second, $\left(\omega_{n, i}^{-1} s\right)^{2} \bar{U}_{i}$, is the torque required to accelerate the motor's mass so as to track the deformation of the elastic element [3]. Only the latter is unique to elastic robots.

Ignoring motor velocity saturation, we can compute performance limits by imposing a symmetric bound on the magnitude of each actuator torque $\left|u_{i}\right| \leq u_{\max , i}$. Introducing

$$
G_{\mathrm{ESP}, i}(s) \triangleq \frac{U_{i}(s)}{\bar{U}_{i}(s)}=1+\left(\omega_{n, i}^{-1} s\right)^{2},
$$

which maps link-side torques to actuator torques, substituting $s=j \omega$ and analyzing its magnitude over $\omega$, provides valuable insight into the performance characteristics of an elastic joint, see Figure 3 (top). Interestingly, the important performance characteristic $G_{\mathrm{ESP}, i}$ is independent of the link inertias or masses. Suppose, we want to interconnect a link impedance $Z_{u, i}=\bar{U}_{i} / V_{i}$ with the $i$ th link. Assuming that the environment acts as an admittance and imposes a link velocity $V_{i}$ on the $i$ th joint, then the corresponding control effort is determined by:

$$
\frac{U_{i}(s)}{V_{i}(s)}=G_{\mathrm{ESP}, i}(s) Z_{u, i}(s) .
$$

In comparison, the control effort for the rigid robot counterpar $\sqrt[4]{4}$, to interconnect a link impedance $Z_{u, i}$, is simply given by $Z_{u, i}$ itself. Comparing (12) with $Z_{u, i}$ allows for the following conclusion. In the frequency range $0<\omega<\sqrt{2} \omega_{n}$, where $\left|G_{\mathrm{ESP}}\right|<1$, the elastic system is superior to the rigid counterpart, in the sense that it requires less control effort to interconnect a link impedance $Z_{u, i}=\bar{U}_{i} / V_{i}$ on the $i$ th link.

\footnotetext{
${ }^{3}$ Considering $\overline{\boldsymbol{u}}_{1}$ as the desired joint torque, the controller achieves global asymptotic convergence $\boldsymbol{\psi} \rightarrow \overline{\boldsymbol{u}}_{1}$ for $t \rightarrow \infty$.

${ }^{4}$ When speaking of the rigid robot counterpart, we refer to the limit case where the joint stiffness values approach infinity.
}
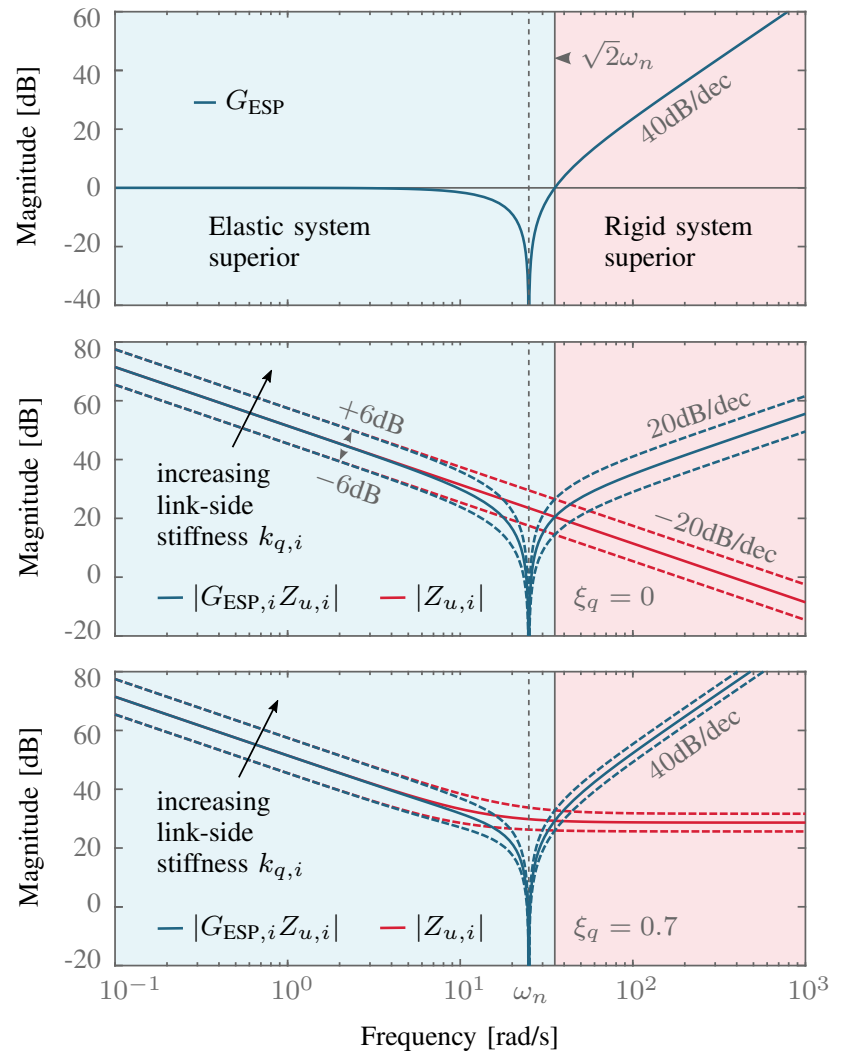

Fig. 3. Top: $G_{\mathrm{ESP}}$ maps the link side torques to the actuator torques. Midbottom: Frequency responses of the control effort transfer functions $G_{\mathrm{ESP}} Z_{u, i}$ and $Z_{u, i}$, which map the $i$ th link velocity to the $i$ th control torque in case of an ASR and rigid robot case, respectively. Mid: implementation of a linear spring. Bottom: implementation of a linear spring and damper.

This property is independent of the particular link impedance choice. This important fact becomes clear considering that the multiplication of two transfer functions, c.f. (12), translates into an addition of their magnitudes responses. A closer inspection of $G_{\text {ESP }}$ reveals that the superiority of the elastic system is maximal for imposed link velocities with a frequency of $\omega=\omega_{n}$. For $\omega>\sqrt{2} \omega_{n}$, the slope of $\left|G_{\mathrm{ESP}}\right|$ is 40 $\mathrm{db} /$ decade and, thus, the elastic system becomes vastly inferior in this frequency range. It is for this reason, that in order to maximize the operational frequency range of an elastic system, it is paramount to minimize the amplitude of $Z_{u, i}$ beyond $\sqrt{2} \omega_{n}$. This aspect is particularly important whenever the environmental admittance imposes velocities with a high frequency content on the $i$ th link.

It is important to underline that the performance analysis results obtained via the ESPi transformation are of general nature and not specific to this particular framework. The $40 \mathrm{~dB}$ rise, in the red zone of Figure 3 is a manifestation of the fact that the joint elasticity acts as a low-pass filter on the actuator output, as highlighted by Pratt in his fundamental work on SEA [3].

\section{A. Implementing Basic Impedance Elements}

Let us compute the control effort required to interconnect linear spring/damper elements to obtain further insights into the performance limits of an ASR. For the $i$ th motor iner- 
tia and joint stiffness, we assume the test bed parameters reported in Table I. Suppose, we want to interconnect a spring element, with spring constant $k_{q, i}$, with the $i$ th link, then $\bar{u}_{1, i}=-k_{q, i} q_{u, i}$ and for the $i$ th link impedance, we have $Z_{u, i}=k_{q, i} / s$. The corresponding frequency responses of $G_{\mathrm{ESP}, i} Z_{u, i}$ and $Z_{u, i}$ are reported in Figure 3 (mid) for the stiffness values $k_{q, i}=\left\{k_{i} / 2, k_{i}, 2 k_{i}\right\}$. Doubling/halving the stiffness $k_{q, i}$ translates into rising/lowering the magnitude of $G_{\mathrm{ESP}, i} Z_{u, i}$ by $6 \mathrm{~dB}$ as indicated by the dashed lines. This becomes immediately clear by inspecting the transfer function. When implementing an additional damper $d_{q, i}=2 \xi_{q} \sqrt{k_{i} b_{i}}$, where $\xi_{q}$ denotes the damping ratio, then, according to the superposition principle, we can simply add up the individual impedances s.t. $Z_{u, i}=k_{q, i} / s+d_{q, i}$. The resulting frequency responses of $G_{\mathrm{ESP}, i} Z_{u, i}$ and $Z_{u, i}$ are reported in Figure 3 , with $\xi_{q}=0.7$ and $k_{q, i}=\left\{k_{i} / 2, k_{i}, 2 k_{i}\right\}$ as above. Clearly, the actuator torque required to implement an additional linkside damper is significantly increased compared to a pure spring implementation in the high frequency regime, where the damper dominates over the spring. Again, the dashed lines indicate a doubling/halving of the imposed stiffness $k_{q, i}$ (the damping factors change accordingly). Inspecting the transfer functions $G_{\mathrm{ESP}, i} Z_{u, i}$ and $Z_{u, i}$, it is straightforward to verify that doubling/halving the stiffness $k_{q, i}$ moves the magnitude plots up/down by $6 \mathrm{~dB}$ in the low frequency domain (springlike behavior) and up/down by $\pm 3 \mathrm{~dB}$ in the high frequency domain (damper-like behavior), c.f. Figure 3 .

\section{IMPROVED DisturbanCE REJECTION VIA DYNAMIC EXTENSIONS}

Building on the insights of the previous two sections, we can formulate damping designs that reduce the control effort in case of an environmental disturbance with high frequency content. Inspired by the design of shock absorbers and considering the observations above, it appears natural to decouple the damper element with a spring-like element to low-pass filter shock loads, thereby greatly reducing the peaks of the interconnection torques $\bar{u}_{i}$ at the moment of impact, see Figure 4 for examples. In fact, choosing a link impedance $Z_{u i}$, with such a decoupled damper, reduces the slope of $\left|G_{\mathrm{ESP}, i} Z_{u, i}\right|$ from $40 \mathrm{~dB} / \mathrm{dec}$ to $20 \mathrm{~dB} / \mathrm{dec}$ in the high frequency regime $\omega>\sqrt{2} \omega_{n, i}$, c.f. Figure 3 (mid) with Figure 3 (bottom). This is a direct consequence of the fact that the interconnection of such elements, via input $\bar{u}_{1, i}$, no longer requires velocity feedback. Consequently, the implementation of (4) no longer requires the critical jerk signals. Impedance elements $Z_{u 1}$ to $Z_{u 4}$ have in common that the damper is decoupled via a spring element (orange), which results in a dynamics extension with one (Variants $1 \& 3$ ) or two additional states (Variants 2 \& 4). Importantly, interconnecting these elements via (4) results in a closed-loop system that can be interpreted as an interconnection of passive Euler Lagrange systems, which again, yields a passive system, see [18] for details.

The dynamic extension $Z_{u 1}$ can be interpreted as the mechanical realization of the popular ,dirty derivatives" filter [8], $Z_{u 2}$ has been initially introduced in [9], [10] and $Z_{u 3}$

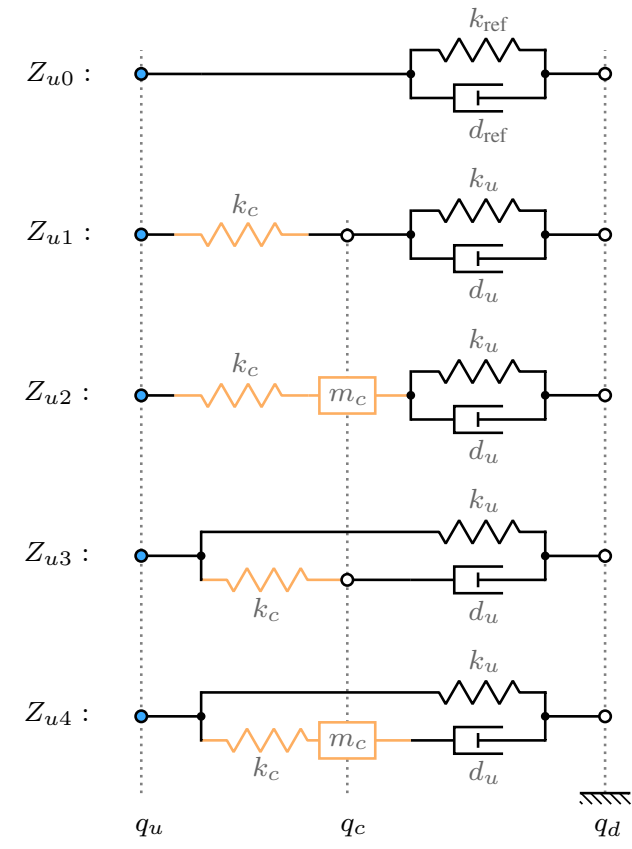

Fig. 4. Mechanical impedance candidates that can be interconnected via the link-side power port to achieve a desired interaction. For Variant 1, the PD element is decoupled via an additional elastic element. In Variant 2, the additional (small) mass guarantees that the dynamic extension represents an actual physical system. Variants 3-4 differ in the sense that only the damper is decoupled via an additional elastic element, thus, the stiffness is uniquely determined by $k_{u}$ analog to $k_{\text {ref }}$ of the reference impedance $Z_{u 0}$.

can be conceived as Hill's muscle model [19]. However, the motivations for these dynamic extensions were different, and thus the recommended gain layouts differ. In case of the dirty derivative filter, the gain $k_{c}$ is usually chosen as high as possible with the intention of approximating the real velocities reasonably well. In [10], [11] the gains are chosen s.t. $k_{c}>>k_{q}$ and $m_{c}<<m$, where $m$ is the link inertia, based on the intuitive reasoning that in this case the two masses tend to become one and the ,felt" stiffness will become $k_{q}$ [10], [20]. In other words, the impedance behavior $Z_{u, 2}$ can be well approximated by the black spring/damper part in Figure 4 When it comes to reducing the actuator torque magnitudes in the moment of a harsh impact, a diametrical layout is desirable. Physical intuition suggests: the lower the coupling stiffness $k_{c}$ the lower the required actuator torque magnitude.

\section{EXPERIMENTS}

A dedicated single joint setup has been used to compare the performance of the enhanced damping design $Z_{u 2}$ to the ,standard" approach $Z_{u 0}$. Figure 5 shows the setup, which consists of a DLR LWR III motor unit in series with a compliant element from DLR C-Runner and a horizontally moving link. The system's dynamics is that of a single elastic joint with a linear spring. To evaluate the closed-loop interaction behavior, the link is equipped with a force sensor (ME KD40s). The link and motor positions are measured with an optical Heidenhain sensor (EBI1023 with 23 bit resolution) and the default LWR III motor sensor. Velocity signals were computed with a standard fourth-order derivative filter with a cut-off frequency of $80 \mathrm{~Hz}$. Link acceleration and jerk signals were computed 


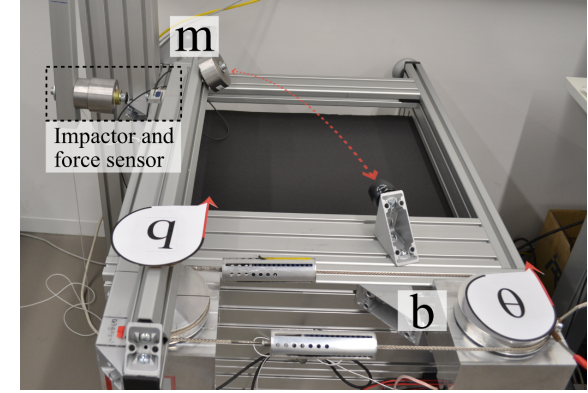

Fig. 5. Experimental setup.

TABLE I

SYSTEM AND CONTROL PARAMETERS

\begin{tabular}{llll}
\hline \multicolumn{2}{c}{ System parameters } & \multicolumn{2}{c}{ Force sensor/control parameters } \\
\hline Link inertia & $1 \mathrm{~kg} \mathrm{~m}^{2}$ & Sensor accuracy & $0.1 \%$ \\
Motor inertia & $0.6 \mathrm{~kg} \mathrm{~m}^{2}$ & Sensor range & $\pm 500 \mathrm{~N}$ \\
Joint stiffness & $374 \mathrm{~N} \mathrm{~m} \mathrm{rad}^{-1}$ & Sensor sample rate & $375 \mathrm{~Hz}$ \\
Actuator limits & $\pm 100 \mathrm{~N} \mathrm{~m}$ & Controller sample rate & $3000 \mathrm{~Hz}$ \\
\hline
\end{tabular}

as discussed in the Appendix. The test bed parameters are summarized in Table [1 and the controller parameters are reported in Table II] The virtual motor was interconnected with a damper, $\bar{u}_{2}=-d_{v} \dot{q}_{v}$, with $d_{v}=2 \xi_{v} \sqrt{b k}$. A factor of $\alpha=1 / 5$ in Table $\Pi$ indicates a reduction of the motor inertia to one fifth of its original value. The motor inertia shaping employed is elaborated in [7]. The dynamic extension parameters were computed with the optimization procedure presented in [21]. The results obtained, c.f. Table [I] minimize the $\mathrm{H}_{\infty}$ norm of the disturbance transfer function $U / \mathcal{Q}_{1}$ under the following constraint: the manipulator impedance, $\mathcal{Q}_{1} / V$, must approximate the reference manipulator impedance defined by the mass-spring-damper system $Z_{\text {ref }}=m s+d_{\text {ref }}+k_{\text {ref }} / s$ with a maximal deviation of $\pm 6 \mathrm{~dB}$, where $m$ is the link inertia, $d_{\text {ref }}=2 \xi_{v} \sqrt{b k_{\text {ref }}}, k_{\text {ref }}=200 \mathrm{~N} \mathrm{~m} \mathrm{rad}^{-1}$. The link impedance obtained from this optimization is plotted in Figure 6 and the parameters are reported in Table II For comparison, $Z_{u 2}^{*}$ with the mass and stiffness ratios $m / m_{c}=10$ and $k / k_{c}=10$ used by [10] is plotted as well. Clearly, the optimization results deviate from the recommendation $m>>m_{c}$ and $k>>k_{c}$ [10]. The reason for this deviation is intuitive, following the arguments from Section $\mathrm{V}$, we know that in order to reduce the control effort the link impedance should fall after $\sqrt{2} \omega_{n}$. The reason why it does not drop immediately after $\sqrt{2} \omega_{n}$ is due to the constraint that the resulting closed-loop behavior must approximate the reference manipulator impedance within $\pm 6 \mathrm{~dB}$.

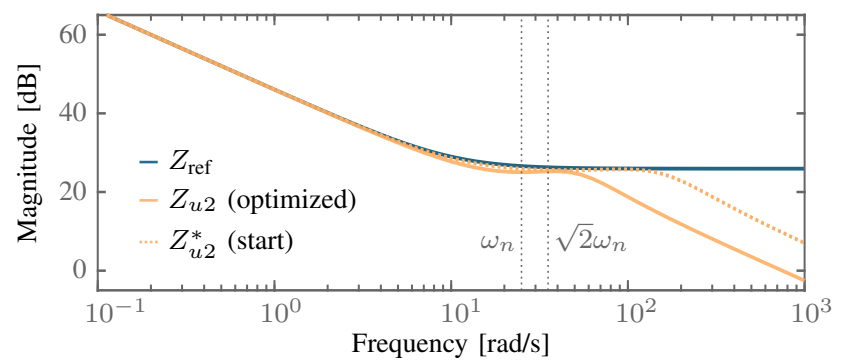

Fig. 6. Frequency responses of the link-side impedance candidates.
TABLE II

CONTROLLER CONFIGURATIONS

\begin{tabular}{|c|c|c|c|c|c|c|c|c|c|}
\hline $\begin{array}{l}\text { Con- } \\
\text { troller }\end{array}$ & $\begin{array}{r}\text { Link } \\
\operatorname{Im}_{]}\end{array}$ & $\begin{array}{l}\text { Motor } \\
\text { lance }\end{array}$ & $\alpha$ & $\begin{array}{r}k_{\mathrm{ref}} \\
{[\mathrm{N}}\end{array}$ & $\begin{array}{c}k_{u} \\
\mathrm{n} \mathrm{rad}\end{array}$ & $\begin{array}{l}k_{c} \\
\left.{ }^{1}\right]\end{array}$ & $\xi_{u}$ & $\xi_{v}$ & $\begin{array}{l}m_{c} \\
{[\mathrm{~kg}]}\end{array}$ \\
\hline $\mathrm{ESP}_{\text {ref }}$ & $Z_{u 0}$ & $Z_{v}$ & 1 & 200 & - & - & 0.7 & 0.3 & - \\
\hline $\mathrm{ESP}_{\mathrm{ref}}^{*}$ & $Z_{u 0}$ & $Z_{v}$ & $1 / 5$ & 200 & - & - & 0.7 & 0.3 & - \\
\hline $\mathrm{ESP}_{2}$ & $Z_{u 2}$ & $Z_{v}$ & 1 & - & 273 & 745 & 0.7 & 0.3 & 0.39 \\
\hline $\mathrm{ESP}_{2}^{*}$ & $Z_{u 2}$ & $Z_{v}$ & $1 / 5$ & - & 273 & 745 & 0.7 & 0.3 & 0.39 \\
\hline
\end{tabular}
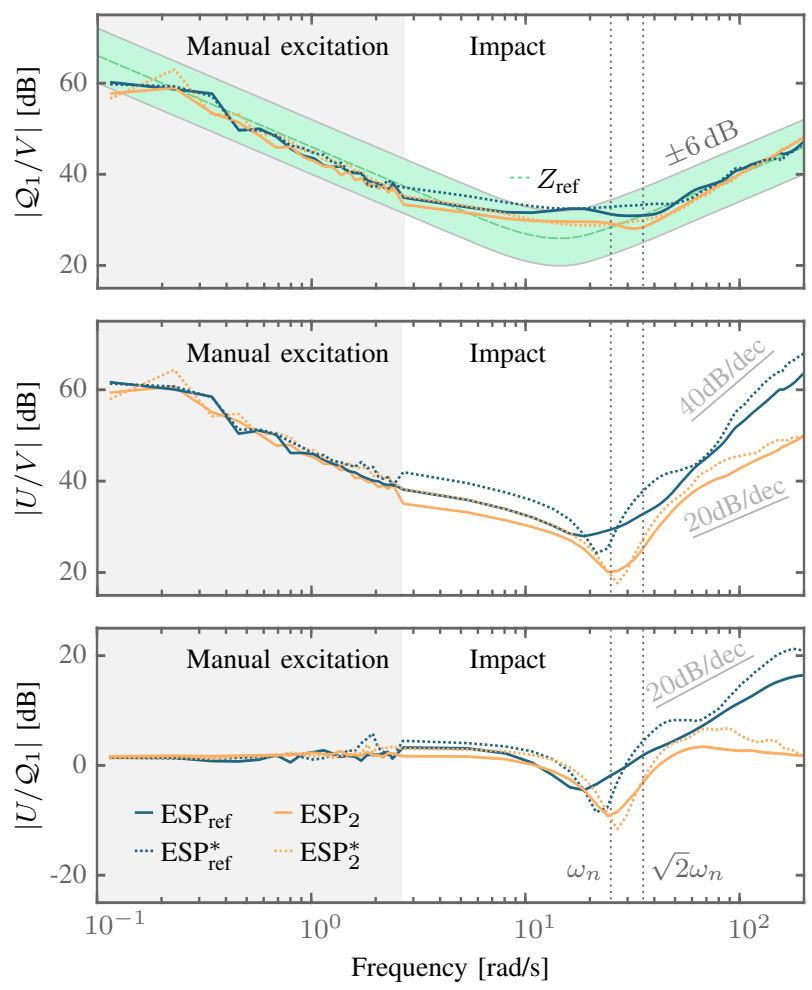

Fig. 7. From top to bottom: frequency response of the manipulator impedance $\mathcal{Q}_{1} / V$, the control efforts $U / V$ and $U / \mathcal{Q}_{1}$.

In the following, three types of experiments are presented. The first one is concerned with the identification of the closedloop manipulator impedance, $\mathcal{Q}_{1} / V$, and the control effort transfer functions $U / V$ and $U / \mathcal{Q}_{1}$, cf. (12). The second one refers to the set-point regulation performance of the control loops. The last one illustrates the performance of the schemes in a basic manipulation task.

\section{A. Experiment 1: Identification of Interaction Behavior}

In this test, a $4 \mathrm{~kg}$ impactor generated a impulse-like excitation of the link, which was followed by a frequency analysis to obtain the frequency responses of the manipulator impedance $\mathcal{Q}_{1} / V$, and the control efforts $U / V$ and $U / \mathcal{Q}_{1}$, see Figure 7 from top to bottom. Clearly, the resulting manipulator impedances for $\mathrm{ESP}_{2}$ and $\mathrm{ESP}_{2}^{*}$ satisfy the tuning goal of lying within a $\pm 6 \mathrm{~dB}$ band (green) of the reference impedance $Z_{\text {ref }}$ (dashed grey curve). Figure 7 further reveals the increase in control effort due to motor inertia shaping.

The effect of the dynamic extension in reducing the control effort in case of high frequency disturbances is clearly visible in the critical frequency range $\left(\omega>\sqrt{2} \omega_{n}\right)$. As predicted by 


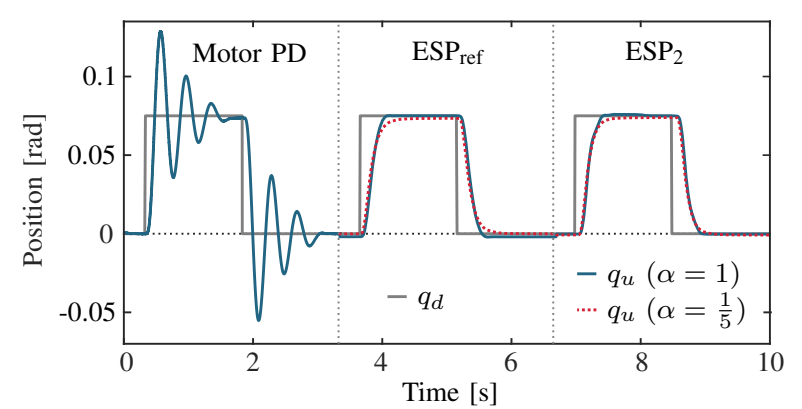

Fig. 8. Set-point regulation behavior.

the performance analysis in Section IV] for controllers with a direct damping injection, $\mathrm{ESP}_{\text {ref }}$ and $\mathrm{ESP}_{\text {ref }}^{*},|U / V|$ exhibits a slope of $40 \mathrm{~dB} / \mathrm{dec}$, whereas for controllers with indirect damping injection, $\mathrm{ESP}_{2}$ and $\mathrm{ESP}_{2}^{*},|U / V|$ exhibits a slope of $20 \mathrm{~dB} / \mathrm{dec}$, see Figure 7 (mid). To check whether these results transfer to the case of external forces $\mathcal{Q}_{1}$ as disturbance input, we identified the frequency response that relates external forces to the control effort, $U / \mathcal{Q}_{1}$, see Figure 7 (bottom). Again, the structural difference between a direct damper and a dynamically decoupled damper implementation is clearly visible past $\omega=\sqrt{2} \omega_{n}$.

\section{B. Experiment 2: Set-Point Regulation}

This experiment is concerned with the analysis of the step response behavior and the results are reported in Figure 8 . The new controllers were evaluated without (blue curve) and with motor inertia shaping (red curve). To eliminate the (slight) overshooting of $\mathrm{ESP}_{2}$ and $\mathrm{ESP}_{2}^{\star}$, we increased the motor damping ratio to $\xi_{v}=0.4$ for this experiment. The performance of the motor PD controller demonstrates the necessity for link-side damping for a system with low intrinsic damping. The marginal steady state error is mostly due to Coulomb friction acting on the link and the elastic mechanism. The slight asymmetry of the steady state error suggests a minor sensor calibration inaccuracy and/or some hysteresis effect. The reduction of the motor inertia reduces the overshooting as physical intuition suggests. Even though link kinetic energy is dissipated only indirectly in the dynamic extension case (underdamped closed-loop system), satisfactory point regulation behaviors can be achieved.

\section{Experiment 3: A Simple Manipulation Task}

Figure 9 reports an experiment involving the interaction of the link with the environment. The desired motion $q_{d}(t)$ follows a sinusoidal trajectory, leading to an unplanned contact with an obstacle. To analyze the worst case contact impact scenario, the link was commanded to impact with a rigid obstacle (end stop). Thus, both impact partners had negligible intrinsic damping properties. The course of the real link position indicates that a transition from a free-space motion to a contact does not cause any stability issue. The energy is properly dissipated and the system is stable in free motion and passive during the entire motion. The total energy of the physical and the virtual (closed-loop) system is reported in Figure 9 , cf. $\mathcal{H}_{q}$ and $\mathcal{H}_{\bar{q}}$. In the latter case, the total energy can

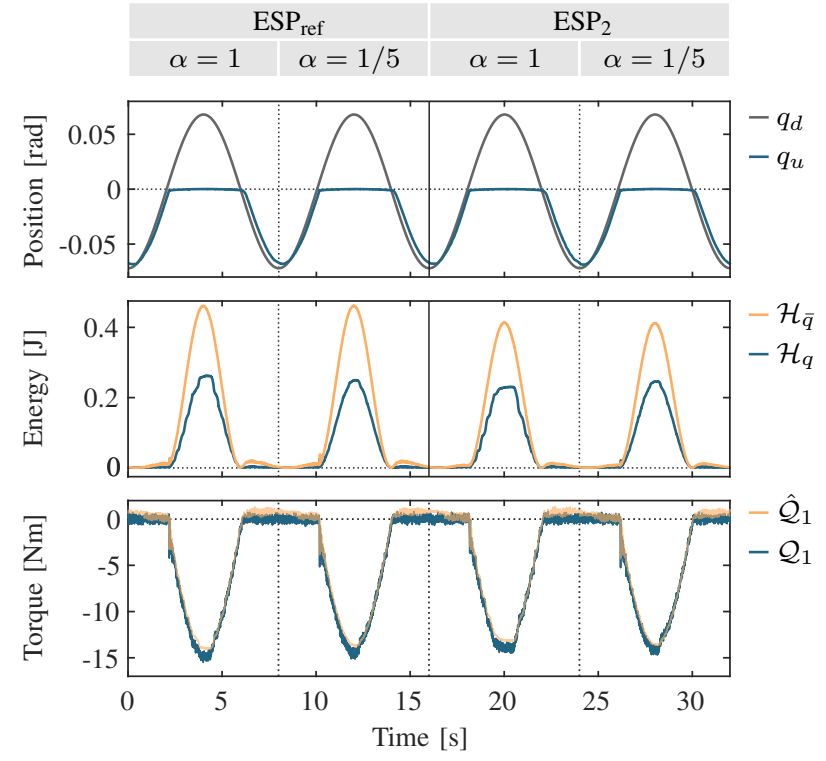

Fig. 9. Top: desired and real position of the link. Mid: real total energy and virtual closed-loop energy. Bottom: measured and estimated external torques $\mathcal{Q}_{1}$ and $\hat{\mathcal{Q}}_{1}$, respectively.

be considered as a storage function for the respective closedloop system and was obtained by summing up all kinetic and potential energies of the virtual system.

Remark. In general, no force sensors are implemented in ASRs and in order to reflect a realistic scenario, we used a momentum based observer [22] instead to estimate the external force to compute link acceleration and jerk signals, via (14) and 15 for experiment 3 . The sensor was only used to report the interaction force.

\section{Monte-Carlo Simulation}

This section reports a Monte Carlo simulation [23] to evaluate the control system robustness with respect to parameter uncertainties. We simulated a single joint with the plant parameters reported in Table $\mathrm{I}$ and considered the same scenario as reported in Experiment 2, with the addition of a step-like force disturbance of $-11 \mathrm{Nm}$ at $t=2 \mathrm{~s}$. A uniform probability density function models the parameter uncertainties, with variations between $\pm 5 \%$ of the nominal values for the link and motor inertias, $m$ and $b$, and $\pm 10 \%$ for the joint stiffness $k$, which is a very pessimistic uncertainty set, c.f. [23]. The control parameters were set as reported in Table [I] with only $\xi_{q}$ changed to 1 since the lack of linkside friction in the simulation necessitates a higher damping ratio to avoid overshooting. The result of 500 runs is plotted in Figure 10. Analyzing the equilibrium conditions for the nominal case, we have that $q-q_{d}=\mathcal{Q}_{1} / k_{r}$, where $k_{r}$ is the resultant closed-loop stiffness, i.e. $k_{\text {ref }}$ for $\mathrm{ESP}_{\text {ref }},\left(\frac{1}{k_{u}}+\frac{1}{k_{c}}\right)^{-1}$ for $\mathrm{ESP}_{1-2}$, and $k_{u}$ for $\mathrm{ESP}_{3-4}$. Let us assume the model parameters are not known exactly, and let $\hat{m}, \hat{b}$ and $\hat{k}$ be the estimates of $m, b$ and $k$ used for the controller implementation. Introducing $\beta \triangleq \hat{k} / k$ and analyzing the equilibrium conditions gives the following relation for the deviation of the equilibrium 


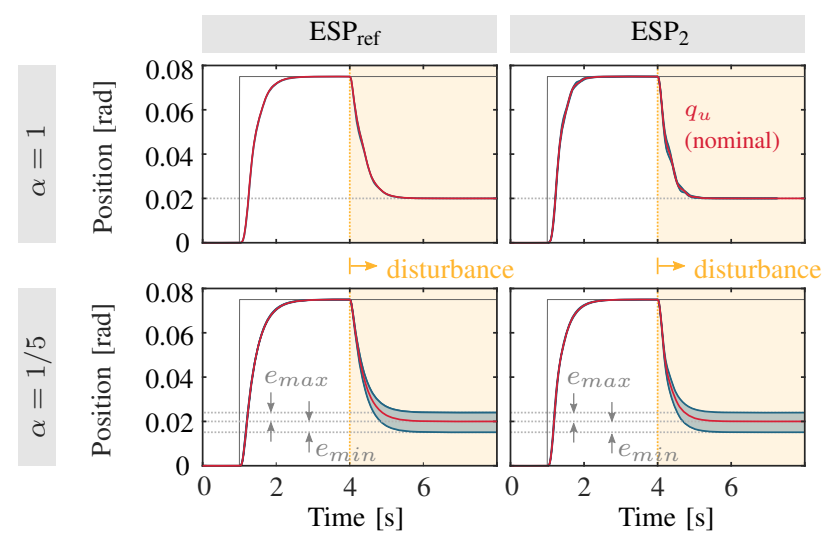

Fig. 10. Monte Carlo simulation: step-response and disturbance rejection.

position from the nominal one:

$$
e=\frac{\mathcal{Q}_{1}}{k_{r}}(1-\alpha)(1-\beta),
$$

which matches the result from the Monte-Carlo simulation, c.f. Figure 10 From (13), we observe that $e$ is non-zero only if $\alpha \neq 1$ and $\beta \neq 1$. Uncertainties in either $m$ or $b$ have no influence on the equilibrium position. The arguments above are no substitute for an in-depth robustness analysis of the proposed designs, and further investigations regarding the stability of an $n$-DoF ASR in the presence of parameter uncertainties are required in future work.

\section{CONCLUSION}

Using the ESPi control framework for ASRs, we analyzed the performance limits arising in the implementation of linkside impedance elements and discussed the challenges concerning link-side damping injection in impact scenarios. These challenges are fundamentally linked to the feedback necessity of the link jerks. Based on the insights obtained, we presented impedance controllers with an enhanced damping design that is based on dynamic extensions. These impedance controllers require fundamentally smaller actuator torques at the moment of impact. Experimental results confirm this favorable behavior compared to classical impedance controllers with a direct damping implementation. As demonstrated on a dedicated test bed, the new impedance designs combine this advantage with the set-point regulation performance of classical impedance controllers. Since the presented impedance controllers are implemented through the ESPi control framework, the system's intrinsic structure is preserved and the resulting closed-loop dynamics are passive and physically intuitive.

\section{APPENDIX}

Using $\boldsymbol{\delta}\left(\boldsymbol{q}, \dot{\boldsymbol{q}}_{u}\right) \triangleq-\boldsymbol{C}\left(\boldsymbol{q}_{u}, \dot{\boldsymbol{q}}_{u}\right) \dot{\boldsymbol{q}}_{u}-\frac{\partial \mathcal{V}(\boldsymbol{q})}{\partial \boldsymbol{q}_{u}}+\mathcal{Q}_{1}$ and the positive definiteness of $\mathcal{M}$, we get obtain from (2) that

$$
\ddot{\boldsymbol{q}}_{u}=\boldsymbol{M}^{-1}\left(\boldsymbol{q}_{u}\right) \boldsymbol{\delta}\left(\boldsymbol{q}, \dot{\boldsymbol{q}}_{u}\right) .
$$

Deriving (14) w.r.t time yields an expression of the link jerks:

$$
\boldsymbol{q}_{u}^{(3)}=\boldsymbol{M}^{-1}\left(\boldsymbol{q}_{u}\right) \dot{\boldsymbol{\delta}}\left(\boldsymbol{q}, \dot{\boldsymbol{q}}_{u}\right)+\dot{\boldsymbol{M}}\left(\boldsymbol{q}_{u}\right) \boldsymbol{\delta}\left(\boldsymbol{q}, \dot{\boldsymbol{q}}_{u}\right)
$$

We see that both the link accelerations and jerks can be written as function of the system states. For the latter case, substitution of (14) into (15) is required.

\section{REFERENCES}

[1] N. Hogan, "Impedance control: An approach to manipulation: Part i-theory," Journal of Dynamic Systems, Measurement, and Control, vol. 107, no. 1, p. 1, 1985.

[2] M. Keppler, D. Lakatos, C. Ott, and A. Albu-Schäffer, "Elastic structure preserving impedance (espi)control for compliantly actuated robots," in 2018 IEEE/RSJ International Conference on Intelligent Robots and Systems (IROS), 2018, pp. 5861-5868.

[3] G. A. Pratt and M. M. Williamson, "Series elastic actuators," in Proceedings 1995 IEEE/RSJ International Conference on Intelligent Robots and Systems. Human Robot Interaction and Cooperative Robots, vol. 1, 1995, pp. 399-406 vol.1.

[4] M. Hutter, C. Gehring, D. Jud, A. Lauber, C. D. Bellicoso, V. Tsounis, J. Hwangbo, K. Bodie, P. Fankhauser, M. Bloesch et al., "Anymala highly mobile and dynamic quadrupedal robot," in 2016 IEEE/RSJ International Conference on Intelligent Robots and Systems (IROS). IEEE, 2016, pp. 38-44.

[5] M. Grebenstein, A. Albu-Schäffer, T. Bahls, M. Chalon, O. Eiberger, W. Friedl, R. Gruber, S. Haddadin, U. Hagn, R. Haslinger, H. Höppner, S. Jörg, M. Nickl, A. Nothhelfer, F. Petit, J. Reill, N. Seitz, T. Wimböck, S. Wolf, T. Wüsthoff, and G. Hirzinger, "The dlr hand arm system," in 2011 IEEE International Conference on Robotics and Automation, 2011, pp. 3175-3182.

[6] B. Vanderborght, A. Albu-Schäffer, A. Bicchi, E. Burdet, D. G. Caldwell, R. Carloni, M. Catalano, O. Eiberger, W. Friedl, G. Ganesh et al., "Variable impedance actuators: A review," Robotics and Autonomous Systems, vol. 61, no. 12, pp. 1601-1614, 2013.

[7] M. Keppler, D. Lakatos, C. Ott, and A. Albu-Schäffer, "Elastic structure preserving (esp) control for compliantly actuated robots," IEEE Transactions on Robotics, vol. 34, no. 2, pp. 317-335, 2018.

[8] R. Kelly, "A simple set-point robot controller by using only position measurements," IFAC Proceedings Volumes, vol. 26, no. 2, pp. 527-530, 1993.

[9] R. Ortega, A. Loria, R. Kelly, and L. Praly, "On passivity-based output feedback global stabilization of euler-lagrange systems," in Decision and Control, 1994., Proceedings of the 33rd IEEE Conference on, vol. 1. IEEE, 1994, pp. 381-386.

[10] S. Stramigioli, "Creating artificial damping by means of damping injection," in In Proceedings of the ASME Dynamic Systems and Control Division, 1996.

[11] G. A. Folkertsma, S. Stramigioli et al., Energy in robotics. Now Publishers, 2017.

[12] A. Loria and R. Ortega, "On tracking control of rigid and flexible joints robots," Appl. Math. Comput. Sci, vol. 5, no. 2, pp. 101-113, 1995.

[13] M. W. Spong, "Modeling and control of elastic joint robots," Transactions of the ASME: Journal of Dynamic Systems, Measurement, and Control, vol. 109, pp. 310-319, 1987.

[14] D. Koditschek, "Natural motion for robot arms," in The 23rd IEEE Conference on Decision and Control. IEEE, dec 1984.

[15] R. Ortega and M. Spong, "Adaptive control of robot manipulators: A tutorial," Automatica, vol. 25, no. 6, pp. 877-888, 1989.

[16] A. van der Schaft, L2-Gain and Passivity Techniques in Nonlinear Control. London New York: Springer, 2017.

[17] C. Ott, Cartesian Impedance Control of Redundant and Flexible-Joint Robots. Springer, 2008.

[18] R. Ortega, Passivity-based control of Euler-Lagrange systems. London New York: Springer, 1998.

[19] A. V. Hill, "The heat of shortening and the dynamic constants of muscle," Proc. R. Soc. Lond. B126136-195, pp. 126-195, 1938.

[20] C. Melchiorri, S. Stramigoli, and S. Andreotti, "Using damping injection and passivity in robotic manipulation," in 1999 IEEE/ASME International Conference on Advanced Intelligent Mechatronics (Cat. No.99TH8399), 1999, pp. 979-984.

[21] D. Wandinger, "Enhancing classical impedance control concepts while ensuring transferability to flexible joint robots," Bachelor Thesis, University of Applied Sciences Munich, September 2020.

[22] A. De Luca, A. Albu-Schäffer, S. Haddadin, and G. Hirzinger, "Collision detection and safe reaction with the dlr-iii lightweight manipulator arm," in 2006 IEEE/RSJ International Conference on Intelligent Robots and Systems. IEEE, 2006, pp. 1623-1630.

[23] L. R. Ray and R. F. Stengel, "A monte carlo approach to the analysis of control system robustness," Automatica, vol. 29, no. 1, pp. 229-236, 1993. 